\title{
基 \\ Influência de diferentes materiais de enriquecimento ambiental na performance reprodutiva de ratos Wistar mantidos em biotério
}

\author{
[Influence of different environmental enrichment materials on reproductive performance of Wistar \\ rats maintained in animal facility]
}

\section{"Artigo Científico/Scientific Article"}

\author{
Ana Caroline Romão da Silva*, Tainara Araújo de Oliveira, Igor Braga Azevedo, Guilherme \\ Nascimento Cunha
}

Centro Universitário de Patos de Minas - UNIPAM, Patos de Minas-MG, Brasil.

*Autora para correspondência/Corresponding author: E-mail: anacrrs@gmail.com

\section{Resumo}

Objetivou-se verificar a influência do enriquecimento ambiental sobre o desempenho reprodutivo de ratos Wistar mantidos em biotério. Doze casais de ratos da linhagem Wistar provenientes do Biotério do Centro Universitário de Patos de Minas foram divididos em quatro grupos com três casais cada: grupo controle (GC) e os grupos com enriquecimento ambiental GEA-1: rolinho de papel, GEA-2: chumaço de algodão e GEA-3: rolinho de papel e chumaço de algodão. Avaliou-se os parâmetros reprodutivos das fêmeas e os índices produtivos das ninhadas. Não foi observado efeito $(p>0,05)$ do enriquecimento ambiental sobre a reprodução dos ratos estudados. Porém, houve uma melhora nas variáveis idade ao primeiro parto, intervalo entre partos, peso ao nascer, taxa de prolificidade e uma diminuição na mortalidade pré-desmame nos grupos enriquecidos. Conclui-se que a utilização de enriquecimento ambiental não influenciou estatisticamente os parâmetros reprodutivos, porém, biologicamente, observou-se aumento da performance nestes parâmetros. O algodão demonstrou superioridade quando comparado aos demais grupos devido à maior facilidade de manuseio do material.

Palavras-chave: bem-estar animal; materiais de nidificação; reprodução; roedores.

\begin{abstract}
The aim of this study was to verify the influence of the environmental enrichment on the reproductive performance of rats kept in an animal facility. Twelve pairs of Wistar rats from the Animal Facility of Centro Universitário de Patos de Minas were divided into four groups with three pairs each: control group (CG) and groups with environmental enrichment GEA-1: paper coil, GEA-2: cotton wad and GEA -3: paper coil and cotton wad. It was evaluated the reproductive parameters of the females and the productive indexes of the litters. No effect ( $p>0.05)$ of environmental enrichment was observed on the reproduction of the studied rats. However, there was an improvement in the variables age at first calving, calving interval, birth weight, prolificity rate, and a decrease in pre-weaning mortality in enriched groups. It was concluded that the use of environmental enrichment did not influence the reproductive parameters statistically, but, biologically, it was observed an increase of the performance in these parameters. The cotton showed superiority when compared to the other groups due to the greater ease of handling of the material.
\end{abstract}

Keywords: animal welfare; nesting materials; reproduction; rodents.

\section{Introdução}

A exposição de animais de experimentação a ambientes enriquecidos há muito tempo tem demonstrado benefícios tanto em animais saudáveis quanto enfermos, jovens ou idosos. Esses ambientes podem elevar os índices 
reprodutivos dos roedores, devido à diminuição do estresse e melhoria do bem-estar, diminuindo a mortalidade, incrementando a taxa reprodutiva, maximizando a relação custo/benefício, melhorando o controle das respostas ansiosas para lidar com o ato da cópula e situações estressantes (Boere, 2001). Segundo Reinhardt e Reinhardt (2006), o uso do enriquecimento ambiental (EA) pode diminuir o índice de infanticídio, canibalismo ou negligência, aumentar a taxa de sucesso de acasalamentos e melhorar o comportamento social com o grupo.

De acordo com Cymerblit-Sabba et al. (2013), Cutuli et al. (2015) e Zuena et al. (2016) a exposição pré-natal ao EA tem efeitos emocionais e hormonais de longo prazo nos descendentes, além disso, esses estudos evidenciaram efeitos benéficos sobre o comportamento e performance cognitiva da prole, especificamente na aprendizagem e memória com indícios de alterações estruturais cerebrais. Baumans e Van Loo (2013) ainda citaram que o EA precoce, ou seja, no pré-desmame diminuiu a ansiedade em ratos adultos a longo prazo, sendo provável que o enriquecimento seja eficaz, pois provocou um aumento no nível de cuidados maternos.

Carvalho et al. (2009) investigaram os efeitos do ambiente enriquecido no desenvolvimento e desempenho reprodutivo de camundongos Swiss em mais de cinco gestações consecutivas por meio do livre acesso dos animais para dispositivos tubulares de diferentes formas e tamanhos. Concluíram que o enriquecimento do meio ambiente não influenciou de forma significativa no tamanho da ninhada, número de animais nascidos vivos, número de animais desmamados por ninhada e peso médio das ninhadas.

Assim, na intenção de otimizar a condição de vida no cativeiro dos roedores, têm-se utilizado ferramentas que promovam o EA (Van de Weerd e Baumans, 1995). Porém, segundo Moreira et al. (2015), mais estudos são necessários para que se obtenha informações suficientes sobre a aplicação deste recurso quando aliado às técnicas de manejo em sistema intensivo de produção. No mercado brasileiro, ainda não existem materiais de enriquecimento com certificação para roedores de laboratório.

Neste contexto, o trabalho teve como objetivo verificar a influência de diferentes materiais de enriquecimento ambiental sobre o desempenho reprodutivo de ratos Wistar pela avaliação de índices reprodutivos das fêmeas e os índices produtivos das ninhadas.

\section{Material e Métodos}

Foram utilizados 12 casais de ratos da linhagem Wistar provenientes do Biotério do Centro Universitário de Patos de Minas UNIPAM, situado em Patos de Minas - MG. O biotério possui sistema de dois corredores (limpo e sujo) entre as salas de experimentação, com fluxo de pessoas e insumos definido e as salas protegidas com barreiras sanitárias (autoclave de barreira, sistemade filtração de ar, diferencial de pressão e air-lock). A temperatura ambiente foi controlada a $22 \pm 2^{\circ} \mathrm{C}$. Possui um sistema de pressão positiva de ar, com insuflação, exaustão e filtração do ar das salas, impedindo a dispersão da amônia no ambiente, realizando 15 a 20 trocas de ar/h e o ciclo de luz é definido 12 horas de claro e 12 horas de escuro. Os ratos foram acondicionados em miniisoladores, contendo um casal em cada, acoplados a uma rack ventilada. A cama utilizada foi de maravalha de pinus e a água fornecida ad libitum. Todo o sistema foi ligado a um gerador, que garantiu sua manutenção em caso de falta de energia elétrica.

Os animais foram divididos em dois grupos. O grupo controle (GC), composto por três casais de ratos, e o grupo com enriquecimento ambiental (GEA), com nove casais sendo este subdividido em três subgrupos contendo três casais cada de acordo com o tipo de enriquecimento. Os grupos foram divididos em: GEA-1: rolinho de papel, GEA-2: chumaço de algodão e GEA-3: rolinho de papel e chumaço de algodão.

Todo o material utilizado para enriquecimento ambiental foi previamente embalado e autoclavado de forma que ficassem estéreis para utilização. Os objetos foram trocados por novos estéreis e rearranjados quanto a sua disposição dentro dos mini-isoladores no momento de sua troca, que ocorreu duas vezes por semana, sempre nos mesmos dias da semana.

Os casais de ratos foram incluídos no estudo com 28 dias de vida (após o desmame) e acompanhados por 10 meses. Neste período, foram mantidos em sistema monogâmico intensivo (Poiley, 1970) e foram observados diariamente, bem como suas proles. A observação de todos os grupos ocorreu sempre no final da tarde e pelo mesmo observador para se evitar estresse dos animais e viés no estudo. O manuseio dos neonatos foi realizado de forma rápida, com cautela, para 
evitar que a mãe diminuísse os seus cuidados. Estes, ainda, foram pesados no primeiro dia de vida, evitando-se o momento de nascimento, quando ainda estavam sujos de sangue. A pesagem foi realizada na balança de precisão (modelo S3201, Bel Photonics, Piracicaba - SP, Brasil) imediatamente após a retirada dos animais do miniisolador. Em seguida, foram devolvidos ao miniisolador friccionando a luva de procedimento na maravalha para que o neonato não ficasse com odor da luva e a mãe não rejeitasse os filhotes. No desmame, aos 28 dias de idade, o procedimento para pesagem foi repetido.

Os seguintes parâmetros foram observados:

a) taxa de fertilidade das matrizes, determinada pela quantidade de cios necessários até $\mathrm{o}$ diagnóstico de uma gestação; b) idade ao primeiro parto; c) intervalo entre partos; d) taxa de prolificidade (número de filhotes nascidos por rata, calculada pelo número de ratas gestantes); e) número de nascidos vivos para cada ninhada; $f$ ) taxa de natimortalidade para cada ninhada (determinada pelo número de nascidos mortos em relação ao número total de nascidos); g) média de peso dos filhotes ao nascer; h) média de peso dos filhotes ao desmame; i) número de animais desmamados para cada fêmea e; j) número de óbitos de filhotes até o desmame (excluindo-se os natimortos).

O delineamento experimental foi inteiramente casualizado com quatro tratamentos e três repetições por trabalho e para avaliação dos resultados foi realizada estatística descritiva para as variáveis taxa de fertilidade e taxa de natimortalidade e o Teste de Tukey com nível de significância de $5 \%(\mathrm{p}<0,05)$ por intermédio do software Assistat para as demais variáveis (idade ao primeiro parto, intervalo entre partos, taxa de prolificidade, número de nascidos vivos para cada ninhada, média de peso dos filhotes ao nascer, média de peso dos filhotes ao desmame, número de animais desmamados para cada fêmea, número de óbitos de filhotes até o desmame).

\section{Resultados e Discussão}

Todos os casais avaliados neste estudo mostraram-se com fertilidade igual a $100 \%$, visto que as fêmeas obtiveram gestação quatro vezes durante o período de acompanhamento, independentemente da idade de acasalamento ou do ambiente a que foram submetidas. A idade média ao primeiro parto foi de 88 dias de vida nos grupos controle e com enriquecimento ambiental utilizando rolinho de papel (GEA-1), enquanto que no grupo com enriquecimento utilizando chumaço de algodão (GEA-2) foi de 93 dias e no grupo enriquecido com chumaço de algodão e rolinho de papel (GEA-3) foi de 99 dias, não apresentando diferença estatisticamente significante $(p>0,05)$.

$\mathrm{O}$ ambiente controlado presente no biotério permite obter bons resultados na reprodução animal, sendo importante salientar que o sucesso desta técnica para roedores em confinamento está estreitamente ligado ao sistema de acasalamento, à questão de segurança sanitária, à funcionalidade e à viabilidade econômica, fato este corroborado por Moreira (2015). Além disso, pode-se considerar que os ambientes enriquecidos podem melhorar o bem-estar de ratos, permitindo que expressem o comportamento natural da espécie pela redução do ócio e possibilitando outras atividades além da sexual, alimentação e autocuidado.

Referente à média do intervalo entre partos, esta foi de $46,8 \pm 13,51$ dias no GC, enquanto que no GEA-1, GEA-2 e GEA-3 foi de $55,1 \pm 2,80$, $56,8 \pm 0,73$ e $53,3 \pm 9,01$ dias, respectivamente. Apesar de não haver diferença estatística significante entre os grupos estudados $(p>0,05)$, o aumento do tempo de intervalo entre partos dos grupos enriquecidos sugeriu que os animais que vivem em ambientes com distrações levam mais tempo para se reproduzir. Este fato é corroborado pelo estudo de Fisch et al. (2017), no qual os

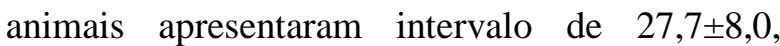
$34,2 \pm 13,3$ e $27,0 \pm 6,3$ dias entre o primeiro e segundo partos nos grupos controle, com tubo PVC e rolinho de papel, respectivamente; apesar de não apresentar diferenças estatisticamente significante $(\mathrm{p}>0,05)$.

O alto valor do desvio padrão do GC e do GEA-3 demonstrou uma grande variação do intervalo entre os partos das fêmeas destes grupos. Isto ocorreu devido a duas fêmeas que imediatamente após o parto apresentaram cio pósparto e obtiveram gestação novamente, tendo duas ninhadas em um curto período de tempo, diferente dos outros grupos, em que as fêmeas gestaram pela segunda vez somente após o desmame, momento em que o macho foi devolvido ao mini-isolador.

A taxa de prolificidade das ratas foi avaliada para todos os tratamentos. Observou-se na Tabela 1 seus valores relativos (\%) nos grupos GC, GEA1, GEA-2 e GEA-3 não apresentando, no entanto, diferenças estatísticas significantes $(p>0,05)$. Carvalho et al. (2009) investigando os efeitos desta técnica sobre o desenvolvimento e o desempenho 
reprodutivo de ratos relataram que o índice de produção do grupo enriquecido foi de $94,1 \pm 14,0 \%$ e o do controle foi de $90,5 \pm 20,0 \%$, indicando que ambos eram similares entre si.

Tabela 1. Média e desvio padrão relativos (\%) da taxa de prolificidade de ratos com ou sem enriquecimento ambiental.

\begin{tabular}{ccc}
\hline Grupo & $\begin{array}{c}\text { Média } \\
\text { Relativa }(\%)\end{array}$ & $\begin{array}{c}\text { Desvio padrão } \\
\text { Relativo }(\%)\end{array}$ \\
\hline GC & 194,0 & 55,15 \\
GEA-1 & 194,0 & 58,17 \\
GEA-2 & 238,6 & 21,92 \\
GEA-3 & 224,6 & 29,40 \\
\hline
\end{tabular}

*GC - grupo controle; GEA-1 - grupo com enriquecimento ambiental 1 (rolinho de papel); GEA-2 - grupo com enriquecimento ambiental 2 (chumaço de algodão); GEA-3 grupo com enriquecimento ambiental 3 (rolinho de papel e chumaço de algodão).

Apesar dos resultados dos grupos do presente estudo não apresentarem diferença estatisticamente significante, observou-se uma diferença biológica no aumento de produtividade destes animais em ambientes melhorados quando comparados com o controle, fato este devido à melhora das condições de bem-estar. Isso ocorreu principalmente nos grupos em que foi utilizado o algodão, pois o material possibilitou maior manipulação e os animais obtiveram maior controle quanto às suas condições de vida.

As médias do índice de natalidade no GC, GEA-1, GEA-2 e GEA-3 foram de 5,83 $\pm 2,73$, $5,83 \pm 2,19, \quad 8,25 \pm 1,87$ e $6,75 \pm 2,20$, respectivamente, sem apresentar, no entanto, diferenças significativas $(p>0,05)$. Não houve presença de natimortos em nenhum dos grupos avaliados. Whitaker et al. (2016) examinaram o efeito da adição de itens em gaiolas de reprodução de ratos de diferentes linhagens (BALB/ cAn-NCrl e 129S2/SvPasCrl) e concluíram que, apesar da superioridade dos ambientes melhorados, não houve diferença estatística significante quanto ao número de filhotes nascidos (em todas as quatro ninhadas) na estirpe BALB/c ou $129 / \mathrm{Sv}$ ou no número de filhotes desmamados na estirpe BALB/c. Já Fisch et al. (2017), que também estudaram os efeitos desta ferramenta no desempenho reprodutivo e na quantidade $\mathrm{e}$ morfologia de complexos cumulus-oócito de Rattus norvegicus, relataram um aumento biológico no número de filhotes nascidos nos grupos enriquecidos, porém a análise estatística não mostrou diferenças significativas $(p>0,05)$ entre os grupos.
No presente estudo, observou-se que o número de filhotes nascidos foi maior nos grupos em que foram utilizadas estratégias de enriquecimento. Essa modificação no ambiente proporcionou melhorias na vida do animal, além de potencializar variações de comportamento, que podem aumentar a produtividade desses animais. Moncek et al. (2004) descreveram reduzida liberação de acetilcolina, cortisona e adrenalina mediante o estresse agudo de animais que viviam em ambiente com enriquecimento ambiental. Os autores relataram, ainda, que esses achados provavelmente foram devido aos animais do ambiente enriquecido estarem submetidos a fatores que sempre os estimulavam, resultando em liberação constante de cortisol, expressando menor variação e uma adaptação mais rápida a estímulos estressantes repentinos.

O peso dos filhotes foi avaliado no primeiro e no $28^{\circ}$ dia de vida (Tabela 2), sem observar diferença estatística significante $(p>0,05)$ entre seus grupos. Este resultado diferiu do encontrado por Van de Weerd et al. (2002) que relataram que os ratos dos grupos enriquecidos apresentavam maior peso do que os de ambientes padrão. De acordo com Hutchinson et al. (2005) e Sirois (2008), os ratos podem responder de maneira insatisfatória à manipulação quando albergados em gaiolas altamente enriquecidas. Isto se deve, provavelmente, ao tamanho reduzido das gaiolas, à necessidade de adaptação destes animais e de rotação periódica ou diferentes estratégias de enriquecimento implementadas simultaneamente.

O ganho de peso dos animais do nascimento ao desmame do grupo GC, GEA-1, GEA-2 e GEA3 teve média de $0,91 \mathrm{~g} / \mathrm{d}, 0,90 \mathrm{~g} / \mathrm{d}, 0,80 \mathrm{~g} / \mathrm{d}$ e 0,84 $\mathrm{g} / \mathrm{d}$, respectivamente. Estes resultados não apresentaram diferença estatística significante $(p>0,05)$ e diferiram do encontrado por Fontes et al. (2012) que relataram que o ganho de peso de camundongos C57BL/6 mantidos em sistema convencional para biotério (open cage) enriquecidos com flocos de algodão foi estatisticamente superior $(\mathrm{p}=0,0102)$ ao grupo padrão.

Um fator determinante na média de ganho de peso dos filhotes foi a ocorrência de canibalismo por uma matriz do grupo GC. Este fato contribuiu significativamente para o aumento de peso dos filhotes remanescentes uma vez que os cuidados maternos foram direcionados apenas para eles, fato este também corroborado por Santos et al. (2010). Além disso, é importante atentar que nos primeiros 
dias de vida os ratos são extremamente dependentes da mãe e necessitam de extensivos cuidados. Dessa forma, ninhadas menores tendem a melhorar a nutrição e desenvolvimento dos filhotes.

A mortalidade pré-desmame deu-se por canibalismo e ocorreu independentemente da existência de enriquecimento ambiental nas gaiolas. No GC ocorreu em 17,14\% dos animais nascidos, enquanto para os GEA-1, GEA-2 e GEA3 a ocorrência foi de 7,14\%, 2,02\% e 17,28\%, respectivamente. $\mathrm{O}$ menor índice de mortalidade pré-desmame ocorreu em ambientes acrescidos com algodão, que pode estar associado à maior possibilidade de estruturação do ambiente por se tratar de um material manipulável e auxiliar na manutenção da temperatura corporal ideal dos filhotes quando utilizados na confecção de ninhos. Interessante destacar que assim como nos outros parâmetros avaliados, neste também não houve diferença estatística significante entre os resultados dos grupos $(p>0,05)$.

Tabela 2. Média de peso ( $\mathrm{g}$ ) dos filhotes no primeiro dia de vida e ao desmame $\left(28^{\circ}\right.$ dia) de ratos com ou sem enriquecimento ambiental.

\begin{tabular}{ccccc}
\hline \multirow{2}{*}{ Grupos } & \multicolumn{2}{c}{ Peso $(\mathbf{g})$ dos filhotes $\left(\mathbf{1}^{\mathbf{0}} \mathbf{~ d i a}\right)$} & \multicolumn{2}{c}{ Peso $(\mathbf{g})$ dos filhotes $\left(\mathbf{2 8}^{\mathbf{0}}\right.$ dia) } \\
\cline { 2 - 5 } & Média & Desvio padrão & Média & Desvio padrão \\
\hline GC & 5,10 & 1,47 & 30,62 & 10,27 \\
GEA-1 & 5,14 & 1,47 & 30,43 & 03,97 \\
GEA-2 & 5,49 & 0,86 & 27,90 & 04,23 \\
GEA-3 & 5,16 & 0,83 & 28,94 & 05,11 \\
\hline
\end{tabular}

*GC - Grupo controle; GEA-1 - grupo com enriquecimento ambiental 1 (rolinho de papel); GEA-2 - grupo com enriquecimento ambiental 2 (chumaço de algodão); GEA-3 - grupo com enriquecimento ambiental 3 (rolinho de papel e chumaço de algodão).

Fontes et al. (2012) inseriram algodão em gaiolas de casais de camundongos C57BL/6, sendo avaliados vários parâmetros reprodutivos e observaram uma redução na mortalidade prédesmame e um aumento no peso de filhotes mantidos em gaiolas abertas enriquecidas com algodão. Enquanto Shair et al. (2012) examinaram como a sobrevivência e o crescimento de filhotes de camundongo C57BL/6 foram afetados pelo uso de algodão, tecidos e iglus de plástico em gaiolas durante a última semana de gestação e os dois primeiros meses após o parto. E concluíram que tanto a presença quanto o tipo de material não influenciaram a sobrevivência e peso dos filhotes.

Os resultados encontrados neste estudo sugeriram que o enriquecimento ambiental influenciou o comportamento materno, resultando em um redirecionamento do gasto de energia das matrizes. Com a construção dos ninhos, a energia que era destinada à produção de calor para a manutenção da temperatura corporal dos filhotes recém-nascidos passou a ser direcionada para outras atividades, sendo, inclusive, mais expressivo no grupo enriquecido com algodão, devido à facilidade na utilização deste material para confeccionar ninhos.

Apesar dos resultados não apresentarem diferenças estatisticamente significativas, os dados mostraram um aumento biológico na taxa de prolificidade das fêmeas, idade ao primeiro parto, intervalo entre partos, média de peso dos filhotes ao nascer e uma diminuição na ocorrência de mortalidade pré-desmame (Figura 1). Estes dados confirmaram que a suplementação ambiental com materiais de nidificação refletiu em uma melhora nas condições de conforto e bem-estar animal.

\section{Conclusão}

A utilização de enriquecimento ambiental não influenciou estatisticamente os parâmetros reprodutivos, porém, biologicamente, observou-se aumento da performance nestes parâmetros. O algodão (GEA-2) demonstrou superioridade quando comparado aos grupos controle e aos enriquecidos com rolinho de papel (GEA-1) e rolinho de papel e algodão (GEA-3) devido à maior facilidade de manuseio do material.

\section{Conflito de Interesse}

Os autores declaram não existir conflito de interesse.

\section{Comitê de Ética}

Este trabalho foi aprovado pelo Comitê de Ética no Uso de Animais do Centro Universitário de Patos de Minas sob número de protocolo: CEUA 03/2017. 


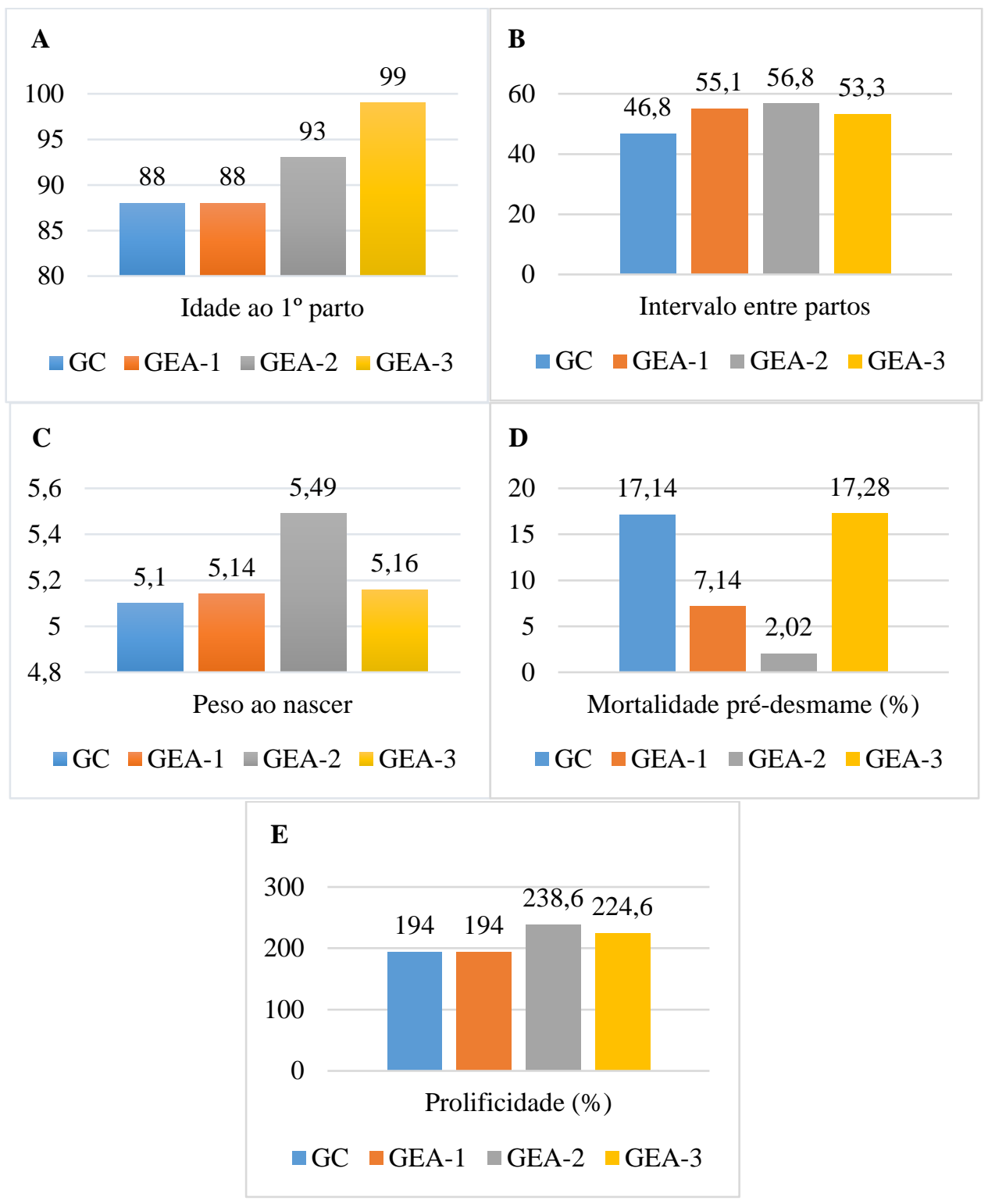

Figura 1. Índices reprodutivos de ratos mantidos em ambientes com ou sem enriquecimento ambiental no biotério. A) Média de idade em dias das fêmeas ao primeiro parto. B) Média do intervalo entre partos (dias). C) Média de peso dos filhotes ao nascer (g). D) Mortalidade pré-desmame (\%). E) Taxa de prolificidade (\%).

\section{Referências}

Baumans, V.; Van Loo, P.L. How to improve housing conditions of laboratory animals: the possibilities of environmental refinement. The Veterinary Journal, 195(1): 24-32, 2013.

Boere, V. Environmental enrichment for neotropical primates in captivity. Ciência Rural, 31(3): 543-551, 2001.

Carvalho, A.F.U.; Araújo, A.J.; Farias, D.F.; Rocha-Bezerra, L.C.B.; Cavalheiro, M.G. Development and reproductive performance of Swiss mice in an enriched environment. Brazilian Journal of Biology, 69(1): 153-160, 2009.
Cutuli, D.; Caporali, P.; Gelfo, F.; Petrosini, L. Prereproductive maternal enrichment influences rat maternal care and offspring developmental trajectories: behavioral performances and neuroplasticity correlates. Frontiers in Behavioral Neuroscience, 9(66): 1-18, 2015.

Cymerblit-Sabba, A.; Lasri, T.; Gruper, M.; AgaMizrachi, S.; Zubedat, S.; Avital, A. Prenatal Enriched Environment improves emotional and attentional reactivity to adulthood stress. Behavioural Brain Research, 241: 185-90, 2013.

Fisch, J.; Oliveira, I.V.; Fank, J.; Paim, L.M.G.; Zandoná, M.R.; Lopes, E.F.; Mello, F.B.; 
Oliveira, A.T.D. Effects of environmental enrichment on reproductive performance and quantity and morphology of cumulus-oocyte complexes obtained from Rattus norvegicus. Theriogenology, 94: 114-119, 2017.

Fontes, R.S.; Santos, R.A.; Ong, F.M.P.; Neves, S.M.P.; Balieiro, J.C.C.; Damy, S.B. Efeito do enriquecimento ambiental na produção de camundongos C57BL/6 mantidos em diferentes sistemas de alojamento. Revista da Sociedade Brasileira de Ciência em Animais de Laboratório, 1: 54-63, 2012.

Hutchinson, E.; Avery, A.; Vande-Woude, S. Environmental enrichment for laboratory rodents. Institute for Laboratory Animal Research Journal, 46(2): 148-160, 2005.

Moncek, F.; Duncko, R.; Johansson, B.B.; Jezova, D. Effect of environmental enrichment on stress related systems in rats. Journal of Neuroendocrinology, 16: 423-431, 2004.

Moreira, V.B; Mattaraia, V.G.M.; Moura, A.S.A.M.T. Lifetime reproductive efficiency of BALB/c mouse pairs after an environmental modification at 3 mating ages. Journal of the American Association for Laboratory Animal Science, 54(1): 29-34, 2015.

Moreira, V.B. Eficiência reprodutiva e comportamento parental de camundongos isogênicos e heterogênicos produzidos em ambiente modificado. Tese (Doutorado) Faculdade de Medicina Veterinária e Zootecnia, Universidade Estadual Paulista, Botucatu - SP, 2015. 106 p.

Poiley, S.M. Animal husbandry and laboratory. Animals Laboratory Animal Care, 20(6):1159-60, 1970.

Reinhardt, V.; Reinhardt, A. Variables, refinement and environmental enrichment for rodents and rabbits kept in research institutions- making life easier for animals in laboratories: Making life easier for animals in laboratories. Washington: Animal Welfare Institute, 2006. 71p.
Santos, M.R.V.; Souza, V.H.; Menezes, I.A.C.; Bitencurt, J.L.; Rezende-Neto, J.M.; Barreto, A.S.; Cunha, F.A.; Marçal, R.M.; TeixeiraSilva, F.; Quíntans-Júnior, L.J.; Barbosa, A.P.O. Parâmetros bioquímicos, fisiológicos e morfológicos de ratos (Rattus novergicus linhagem Wistar) produzidos pelo Biotério Central da Universidade Federal de Sergipe. Scientia Plena, 6(10): 1-6, 2010.

Shair, H.N.; Nunez, Y.; Osman, M.M. Enrichment materials do not negatively affect reproductive success and offspring survival and weight in mice. Lab Animal, 41(1): 14-9, 2012.

Sirois, M. Medicina de animais de laboratório: princípios e procedimentos. São Paulo: Roca, 2008. 332 p.

Van de Weerd, H.A.; Baumans, V. Environmental enrichment in rodents. In: Smith, C.P.; Taylor, V. Environmental Enrichment Information Resources for Laboratory Animals: 19651995. England: AWIC Resource Series, 2, 1995. p. 145-149.

Van de Weerd, H.; Aarsen, E.L.; Mulder, A.; Kruitwagen, C.L.J.J.; Hendriksen, C.F.M.; Baumans, V. Effects of environmental enrichment for mice: variation in experimental results. Journal of Applied Animal Welfare Science, 5(2): 87-109, 2002.

Whitaker, J.W.; Moy, S.S.; Pritchett-Corning, K.R.; Fletcher, C.A. Effects of enrichment and litter parity on reproductive performance and behavior in BALB/c and 129/Sv mice. Journal of the American Association for Laboratory Animal Science, 55(4): 387-399, 2016.

Zuena, A.R.; Zinni, M.; Giuli, C.; Cinque, C.; Alemà, G.S.; Giuliani, A.; Catalani, A.; Casolini, P.; Cozzolino, R. Maternal exposure to environmental enrichment before and during gestation influences behaviour of rat offspring in a sex-specific manner. Physiology \& Behavior, 163: 274-287, 2016. 